Check for updates

Cite this: RSC Adv., 2019, 9, 11101

Received 26th February 2019

Accepted 28th March 2019

DOI: $10.1039 / c 9 r a 01443 h$

rsc.li/rsc-advances

\section{Microfluidic preparation, shrinkage, and surface modification of monodispersed alginate microbeads for 3D cell culture}

\author{
Dan Yu, $\dagger^{\mathrm{ab}}$ Ziye Dong, (D) $\dagger^{\mathrm{b}}$ HyunTaek Lim, ${ }^{\mathrm{b}}$ Yuting Chen, ${ }^{\mathrm{c}}$ Zhenya Ding, (D) ${ }^{\mathrm{b}}$ \\ Nadia Sultana, ${ }^{b}$ Jiangyu Wu, ${ }^{c}$ Bingyu Qin, ${ }^{a}$ Jianjian Cheng*a and Wei Li (iD *b
}

Functionalized alginate microbeads (MB) have been widely used for three-dimensional (3D) culture of cells and creating biomimetic tissue models. However, conventional methods for preparing these MB suffer from poor polydispersity, due to coalescence of droplets during the gelation process and post-aggregation. It remains an immense challenge to prepare alginate MB with narrow size distribution and uniform shape, especially when their diameters are similar to the size of cells. In this work, we developed a simple method to produce monodispersed, cell-size alginate MB through microfluidic emulsification, followed by a controlled shrinkage process and gelation in mineral oil with low concentration of calcium ion $\left(\mathrm{Ca}^{2+}\right)$. During the gelation process caused by the diffusion of $\mathrm{Ca}^{2+}$ from the oil to water phase, a large amount of satellite droplets with sub-micrometer sizes was formed at the water/oil interface. As a result, each original droplet was transformed to one shrunken-MB with much smaller size and numerous submicron-size satellites. To explore the feasibility of the shrunken-MB for culturing with cells, we have successfully modified a variety of polymer nanofilms on MB surfaces using a layer-by-layer assembly approach. Finally, the nanofilm-modified MB was applied to a 3D culture of GFP-expressing fibroblast cells and demonstrated good biocompatibility.

\section{Introduction}

The wide-ranging and growing applications of cell aggregates incorporating polymeric microbeads (MB) have established both the utility and promise of the ex vivo three dimensional (3D) cell culture platform to mimic native tissue microenvironments. ${ }^{1}$ A variety of biomaterials have been explored for fabricating cell-associated $\mathrm{MB}$, where selected materials are most generally non-toxic and elicit limited inflammatory or immunological responses. ${ }^{1,2}$ Alginate (ALG) MB have been used extensively for $3 \mathrm{D}$ cell/MB co-culture, owing to advantages including biocompatibility, biodegradability and low cost. ${ }^{\mathbf{1 - 4}}$ Currently, many methods have been developed for generation of ALG MB with sizes ranging from 10 to $300 \mu \mathrm{m}$, including grinding, electrodispersion, spray-drying, and emulsification. ${ }^{5-11}$ Among these methods, emulsification is the most common approach for fabrication of $\mathrm{MB}$ because of its

${ }^{a}$ Department of Critical Care Medicine, People's Hospital of Zhengzhou University (Henan Provincial People's Hospital), Zhengzhou, 450003, China. E-mail: chengjian030701@163.com

${ }^{b}$ Department of Chemical Engineering, Texas Tech University, 807 Canton Ave, Lubbock, Texas, 79409, USA. E-mail:wei.li@ttu.edu

${ }^{c}$ School of Materials Science \& Engineering, Wuhan Institute of Technology, LiuFang Campus, No. 206, Guanggu 1st Road, Donghu New \& High Technology Development Zone, Wuhan, 430205, P. R. China

$\dagger$ Co-first author. simplicity: aqueous solution of sodium alginate is dripped into an aqueous solution containing calcium ions $\left(\mathrm{Ca}^{2+}\right)$ that can induce gelation by physical crosslinking of mannuronic acid in the ALG chains. ${ }^{3,4}$ However, the final ALG MB normally has poor polydispersity due to coalescence of ALG droplets during the gelation process and post-aggregation of ALG MB. It is inherently challenging to prepare ALG microbeads with narrow size distribution and uniform shape.

Microfluidic emulsification is a simple and rapid method to prepare monodispersed ALG droplets with well-controlled shapes and sizes, and the resulting ALG MB can be produced by crosslinking the droplets with $\mathrm{Ca}^{2+}$ simultaneously or afterwards. ${ }^{12-18}$ Ideally, MB with a size similar to cells (10-20 microns) are desired for $3 \mathrm{D}$ cell/MB co-culture because of their potential to mimic cell-cell interactions as 'synthetic neighbors' ${ }^{19}$ Also, recent study demonstrated that surface curvature can differentially regulates stem cell migration and differentiation as it affects cells attachment morphology and induces nuclear deformation. ${ }^{20}$ However, generating monodisperse MPs in 10-20 microns range poses specific challenges as it is generally larger than that obtained through typical emulsionbased techniques and smaller than particles fabricated through most mold-based techniques. ${ }^{1}$ Furthermore, most microfluidic devices encounter difficulties in generating beads or particles in the cell-size range, which is due to the size dependence between the microfluidic channel and the 
produced droplets. Such small microchannels are not only hard to fabricate but also suffer from clogging issues. ${ }^{21}$ So far, a few efforts have been devoted to making small alginate $\mathrm{MB}$ from microfluidic devices. ${ }^{21-24}$ In one study, ALG droplets were generated in a regular microfluidic device and collected on an agarose slab, where ALG droplets shrank and were crosslinked by $\mathrm{Ca}^{2+}$ to achieve designed sizes. ${ }^{22}$ However, the addition of the agarose slab raises the complexity of fabrication process.

In this work, we developed a simple method to produce cellsize ALG MB through microfluidic emulsification followed by a controlled shrinkage process (Fig. 1a). First, we generated aqueous ALG droplets with a size of 40-110 microns in a microfluidic device using mineral oil with a low concentration of $\mathrm{Ca}^{2+}$ as a continuous phase. ${ }^{13,25,26}$ Then the generated droplets were collected from the outlet of the microfluidic devices, maintained in the continuous phase and allowed in situ gelation to form ALG MB. During the slow gelation process caused by diffusion of $\mathrm{Ca}^{2+}$ from oil to water phase, a large amount of satellite ALG droplets with sub-micrometer sizes were formed at the water/oil interface. As a result, each ALG droplet was transformed to one shrunken-MB 5-30 microns in size and with numerous submicron-size satellites. After completion of gelation, shrunken-MB was separated through multi-step washing to remove oil and satellites. Furthermore, to test the feasibility of applying the ALG shrunken-MB for cell/MB co-culture, the surface of ALG MB was successfully modified with a variety of nanofilms through a layer-by-layer assembly approach (Fig. 1b). Finally, the nanofilm-modified ALG MB was applied to a 3D culture of GFP-expressing fibroblast cells (NIH-3T3-eGFP) and demonstrated good biocompatibility.

\section{Experimental}

\section{Materials}

Alginic acid sodium salt from brown algae (ALG), polyallylamine hydrochloride (PAH, $\left.M_{\mathrm{w}}=65000\right)$, poly-t-arginine (PARG) $\left(M_{\mathrm{w}}=\right.$ 15 000; 15 000-70 000; >70 000), calcium iodide, Span 80, Triton $\mathrm{X}-100$, mineral oil, alginate lyase, and other chemicals were purchased from Sigma-Aldrich. Silicon wafers, biotin, and Avidin-Texas Red conjugate were purchased from ThermoFisher Scientific. Polydimethylsiloxane elastomer (PDMS) (Sylgard 184) was provided by Dow Corning. Fluorescein labeled PAH (FPAH) was synthesized using published protocols. ${ }^{27} \mathrm{SU}-8-2050$ negative photoresist and SU-8 developer were obtained from MicroChem.

\section{Device preparation}

The flow focusing microfluidic devices were fabricated by standard lithograph method. ${ }^{26}$ Specifically, the desired features were drawn by AutoCAD and printed on transparent films as masks. Then, SU-8-2050 was spin-coated on a silicon wafer. After softbaking, exposure to mask, and post-baking, the unexposed epoxy part was dissolved by SU-8 developer to obtain the final master. The height of the master is approximately 100 microns. To fabricate the PDMS replica, PDMS with $10: 1$ ratio of monomer to curing agent was mixed vigorously and poured on the SU-8 master. After $30 \mathrm{~min}$ de-gas and heating at $65{ }^{\circ} \mathrm{C}$ for $2 \mathrm{~h}$, the cured PDMS layer was detached from the SU-8 master. The final devices were made by bonding the PDMS replica and a flat PDMS slab after plasma treatment for $45 \mathrm{~s}$. Devices were allowed to recover to hydrophobic surface for 1 week at room temperature.

\section{(a)}

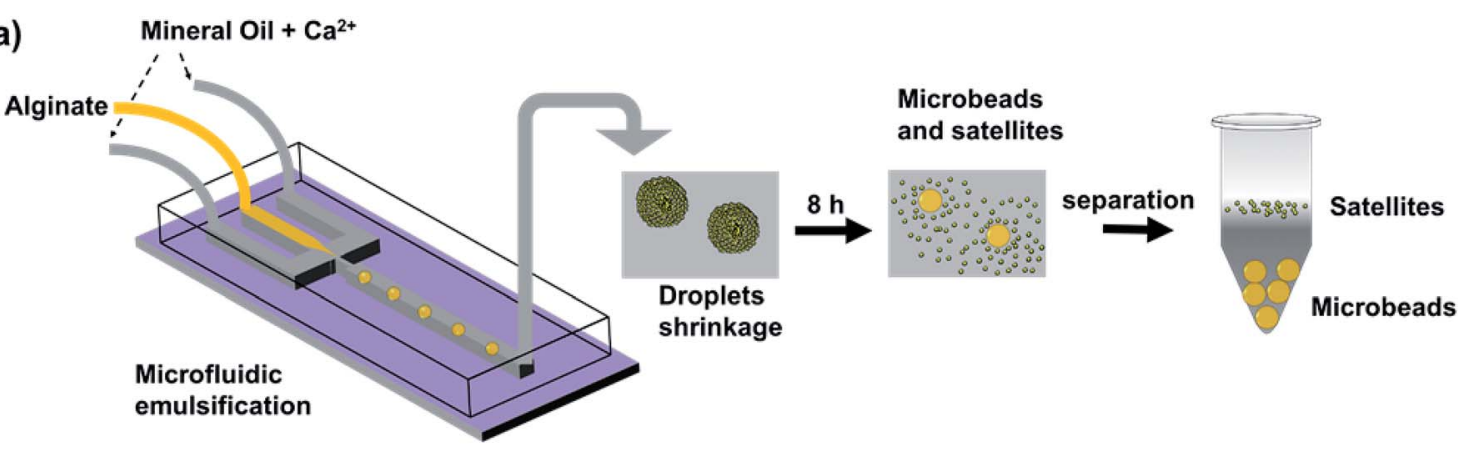

\section{Washing}

(b)

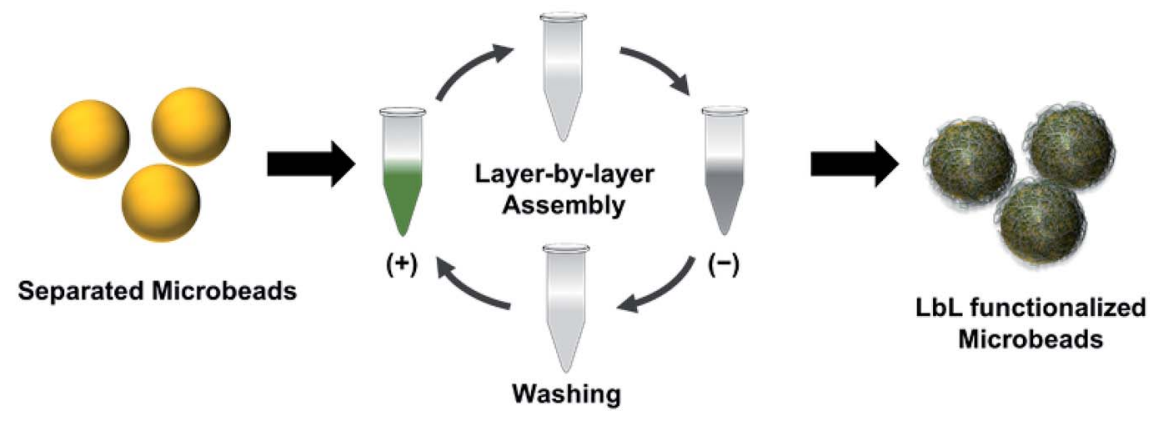

Fig. 1 Schematics of microfluidic emulsification of ALG droplets in mineral oil containing $\mathrm{Ca}^{2+}(\mathrm{a})$, followed by droplet shrinkage process and separation of MB from satellites by washing and centrifugation; and (b) layer-by-layer (LbL) assembly of multilayered nanofilm on the surface of separated ALG MB. 


\section{Droplet generation}

ALG was dissolved in DI water to form an aqueous solution with desired concentration. Calcium iodide $0.1 \mathrm{wt} \%$ and Span 80 $4 \mathrm{wt} \%$ were dissolved in mineral oil as oil phase. After filtering with $5 \mu \mathrm{m}$ filter, aqueous and oil solutions were loaded in separate syringes. Meanwhile, a flow focusing microfluidic device was connected to the syringes by Tygon tubing. The oil phase was first introduced into the microfluidic device through two side channels. Afterward, the ALG solution was introduced into the microfluidic device via the center channel. Droplets were constantly formed after the ALG solution went through the orifice of microfluidic device and were collected in a centrifuge tube at the outlet of the microfluidic device.

\section{Droplet shrinkage observation}

The droplet generation setup was similar to the above process in which BALG and Avidin Texas Red $\left(0.05 \mathrm{mg} \mathrm{mL}^{-1}\right)$ mixture was used instead of ALG solution. In order to observe the shrinkage process in situ, a glass slide was used to collect one drop of fluid (approximate $50 \mu \mathrm{L}$ ) from the outlet of droplet generation device and the droplet was observed under an Olympus BX53 fluorescence microscope. Fluorescent and bright field images were taken manually at certain interval for $8 \mathrm{~h}$ after the droplet generation.

\section{Droplet washing}

$24 \mathrm{~h}$ after droplet generation, the collected ALG beads were washed to get rid of any oil contamination. Specifically, ALG beads were spun down by $200 \mathrm{~g}, 5 \mathrm{~min}$ centrifuge before supernatant was removed by pipet. Then $1 \mathrm{wt} \%$ Triton X 100 water solution was introduced in the centrifuge tube. The process was repeated 3 times, then DI water was used to wash the ALG beads 3 times to take out Triton X 100. The washed ALG beads were stored at room temperature for further study.

\section{Preparation of LbL film on ALG microbeads}

LbL films were modified on to ALG microbeads according to previous published works. ${ }^{28,29}$ Briefly, PARG, FPAH and ALG were dissolved in DI water at a concentration of $2 \mathrm{mg} \mathrm{mL}^{-1}$. To perform LbL process, the prepared ALG beads were alternatively incubated with positively charged polymer solutions (FPAH or PARG) or negatively charged polymer solution (ALG) for $5 \mathrm{~min}$ with washing steps in between. The process was repeated 5 times.

\section{Film thickness measurements}

LbL films were built by the capillary flow LbL method to test film thickness as described in our previous work. ${ }^{30}$ Briefly, a silicon wafer was air plasma treated for $5 \mathrm{~min}$. Then a PDMS slab with straight channel feature was bonded on the silicon wafer after air plasma treatment for $50 \mathrm{~s}$ together. Afterwards, PARG or ALG solutions were introduced in the microfluidic channel alternatively for $5 \mathrm{~min}$ with washing steps in between until the process was repeated the desired number of times.
Finally, the PDMS slab was peeled off and LbL film was measured by an optical profilometer (Dektak XT).

\section{Degradation of nanofilm on the surface of ALG microbeads}

ALG lyase was dissolved in PBS at $1 \mathrm{mg} \mathrm{mL}^{-1}$ and incubated at $37^{\circ} \mathrm{C}$ for $20 \mathrm{~min}$ as enzymatically degradation solutions, which were always made within $1 \mathrm{~h}$ before degradation experiments. Texas Red labeled ALG beads were incubated with the freshly made ALG lyase solution for $10 \mathrm{~min}$ at room temperature and washed by PBS. Fluorescent images were taken before and after the degradation process. ImageJ was used for image analysis.

\section{Scanning electron microscopy (SEM)}

ALG beads were dried at room temperature in a chemical hood for $24 \mathrm{~h}$. After being coated by a sputter coater for $90 \mathrm{~s}$, the beads were scanned under SEM ( $2 \mathrm{kV}$ and 1 brightness) at 200, 1500, and 5000 magnifications.

\section{Transmission electron microscopy (TEM)}

ALG beads were fixed with epoxy resin and sliced by microtome for TEM. Specifically, the ALG beads were incubated in 100\% acetone for $10 \mathrm{~min}$ followed by $10 \%$ epoxy resin acetone solution for $1 \mathrm{~h}$. Then the beads solution was poured into a mold with additional resin used to fill the mold. After baking at $70{ }^{\circ} \mathrm{C}$ for $48 \mathrm{~h}$, the bead-imbedded epoxy was sliced into layers and transferred onto a copper grid (100 mesh). The sample loaded grid was incubated with $4 \%$ uranyl acetate for $10 \mathrm{~min}$ to achieve higher TEM resolution. After washing with DI water, the sample was characterized by Hitachi H-8100 TEM.

\section{Cell culture and counting}

A reporter cell line, NIH-3T3-eGFP, was purchased from Cell Biolabs Inc. Cells were cultured at $37^{\circ} \mathrm{C}$ incubator $\left(5 \% \mathrm{CO}_{2}\right.$ and 95\% air) in Dulbecco's Modified Eagle's Medium (DMEM) base cell culture medium with 10\% fetal bovine serum (FBS) and 1\% of penicillin-streptomycin. Cell medium was changed every 2 days and cells were sub-cultured when the cells reached $70 \%$ confluency.

\section{Cell culture with ALG microbeads}

ALG MB with 5 bilayers of BALG/PARG were sterilized by incubating with $70 \%$ ethanol for $12 \mathrm{~h}$. Afterwards, the beads were washed by cell culture medium 5 times and stored at $4{ }^{\circ} \mathrm{C}$ until cell culture. NIH-3T3-eGFP cells were detached by contacting with $0.25 \%$ trypsin EDTA for $5 \mathrm{~min}$ and neutralized by cell culture medium. Finally, 100000 per mL cells and 400000 per $\mathrm{mL}$ ALG beads were mixed and cultured in a 96-well plate at $37{ }^{\circ} \mathrm{C}$.

\section{Results and discussion}

\section{Preparation of ALG droplets and MB}

A flow-focusing microfluidic device was used to continuously form ALG droplets Fig. 2a. ALG solution was injected into a 40 $\mu \mathrm{m}$ width orifice and broken into individual droplets with 
(a)

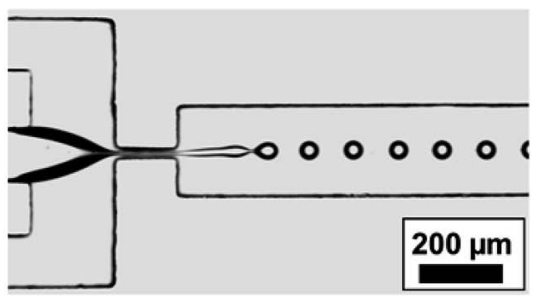

(c)

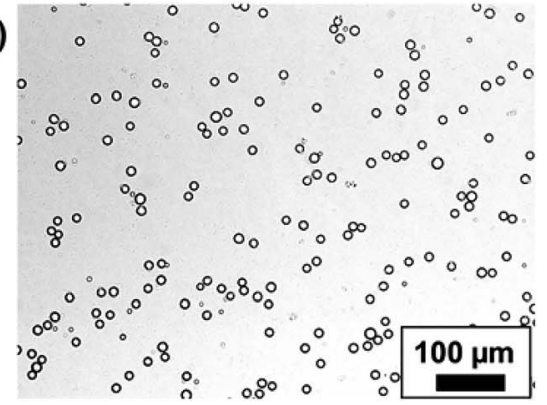

(b)

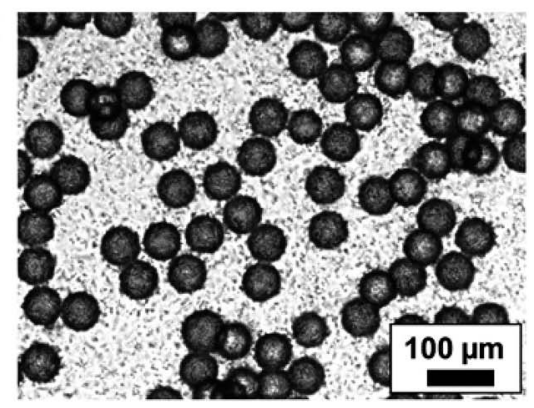

(d)

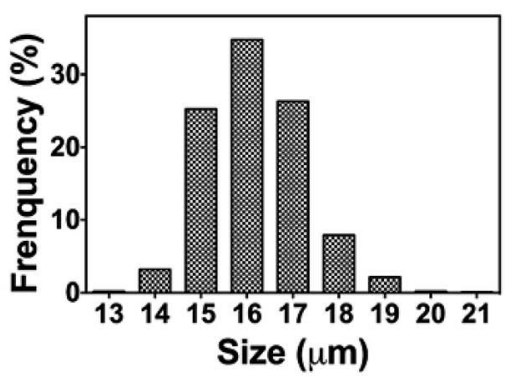

Fig. 2 (a) A typical microscopy image of ALG droplet (2 wt\%) generation in a flow focusing microfluidic device; (b) microscopy image of ALG droplets (2 wt\%) collected at the outlet of generation device; (c) microscopy image of solidified ALG shrunken-MB in mineral oil at $8 \mathrm{~h}$ after generation, and the submicron-size satellites are invisible under optical microscope; (d) size distribution of the ALG shrunken-MB connected after washing and removal of the satellites.

uniform size distribution. In the system, mineral oil with $0.1 \mathrm{wt} \% \mathrm{CaI}_{2}$ and $4 \mathrm{wt} \%$ Span 80 was employed as continuous phase. Span 80, a nonionic surfactant, stabilized ALG droplets and prevented the coalescence of droplets, while $\mathrm{Ca}^{2+}$ ions crosslinked and solidified the ALG droplets. Initial droplet sizes of 40,70 and $110 \mu \mathrm{m}$ were selected to achieve various sizes for the final shrunken-MB. The flow rates for ALG phase and oil phase were ranging from 0.2 to $0.4 \mathrm{~mL} \mathrm{~h}^{-1}$ and from 0.6 to $1.0 \mathrm{~mL} \mathrm{~h}^{-1}$, respectively. As shown in previous reported work, ${ }^{25,31}$ droplet sizes can be easily controlled by the flow rates (and ratio) of ALG solution phase and the mineral oil phase. It is noted that other initial droplet size in the range of 30-200 $\mu \mathrm{m}$ can be also be achieved. In order to examine the gelation process in a real-time fashion, ALG droplets were collected in a flat chamber at the outlet and observed under microscope (Fig. 2b). The just generated ALG droplets showed uniform size and approximately smooth surface (Fig. 2a). However, after 2 min incubation with $\mathrm{Ca}^{2+}$ ion containing mineral oil, the ALG droplets' surface turned to a grain and buckling structure. A similar phenomenon was observed in the previously reported work. ${ }^{13}$ It is noted that the gelation of ALG droplets was a diffusion-controlled process where $\mathrm{Ca}^{2+}$ ions move from the oil phase to aqueous phase, and triggered the crosslinking of ALG at the surface of droplets to form a "skin" layer, then $\mathrm{Ca}^{2+}$ ions further diffused towards the center of ALG droplets. The process of gelation took place in oil for $8 \mathrm{~h}$, during which the skin layer broke and formed numerous submicron size satellites, while the original droplet with diameter of $40 \mu \mathrm{m}$ shrunken into a smaller-sized and solidified ALG MB with a final size of $16 \pm 2 \mu \mathrm{m}$ (Fig. $2 \mathrm{c}$ and d).

\section{Shrinkage of ALG droplets}

We further monitored the shrinkage and gelation process of the ALG droplets (Fig. 3a). At $t=0$ min where ALG droplets were collected from the microchip (approximately 10 seconds from droplets are generated from the orifice), the droplet surface was slightly cloudy and bumpy, which suggested the gelation already had started. In the following $2 \mathrm{~h}$, the surface of ALG droplets became irregular, fuzzy, and hairy, as submicron-size satellites were formed by breaking the skin layer of ALG and precipitated in the oil phase. From $t=2 \mathrm{~h}$ to $8 \mathrm{~h}$, the fuzzy satellites gradually dissipated in the surrounding oil due to Brownian motions, and finally became invisible under optical microscope; the shrunken-MB remained in relatively the same location and contained a generally smooth surface. As shown in Fig. $3 \mathrm{~b}$, the size of ALG droplets and the resulting shrunken-MB decreased linearly for the first $5 \mathrm{~h}$ and stayed relatively constant. Additionally, we generated Texas Red-labeled ALG droplets and monitored their shrinkage behavior under microscope. As shown in Fig. $3 \mathrm{c}$ and d, the initial droplets were approximate $40-42 \mu \mathrm{m}$ in diameter and the Texas Red fluorescence was colocalized with the ALG droplets, indicating that dye-labeled ALG was only presented in the aqueous droplets and not in the oil phase. Nevertheless, after 60 min incubation in mineral oil containing $\mathrm{Ca}^{2+}$ ions, the Texas Red fluorescent signal spread to entire oil phase, which may due to: (1) diffusion of dye-labeled ALG molecules into the oil phase, and (2) formation of ALG satellite droplets and submicron size ALG MB that were dissipated in the oil phase.

We also explored the droplet generation experiment by forming droplets at different initial sizes with different ALG 
(a)

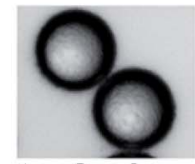

$\mathbf{t}=\mathbf{0} \min$

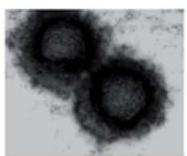

$t=240 \min$

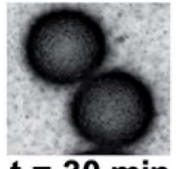

$\mathbf{t}=\mathbf{3 0} \mathrm{min}$

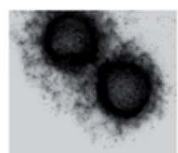

$\mathbf{t}=\mathbf{3 0 0} \min$
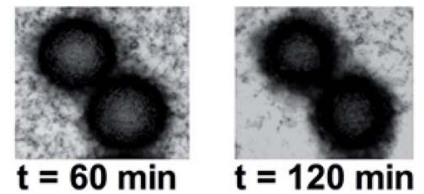

$t=120 \min$
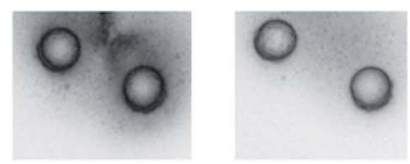

$t=360 \min t=420 \mathrm{~min}$
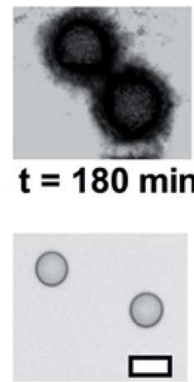

$t=480 \min$ (b)

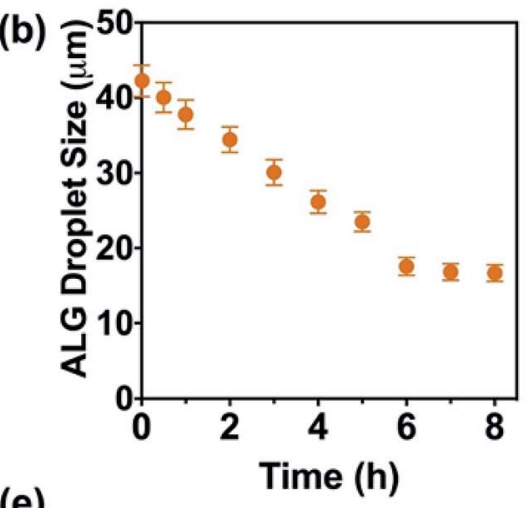

(e)
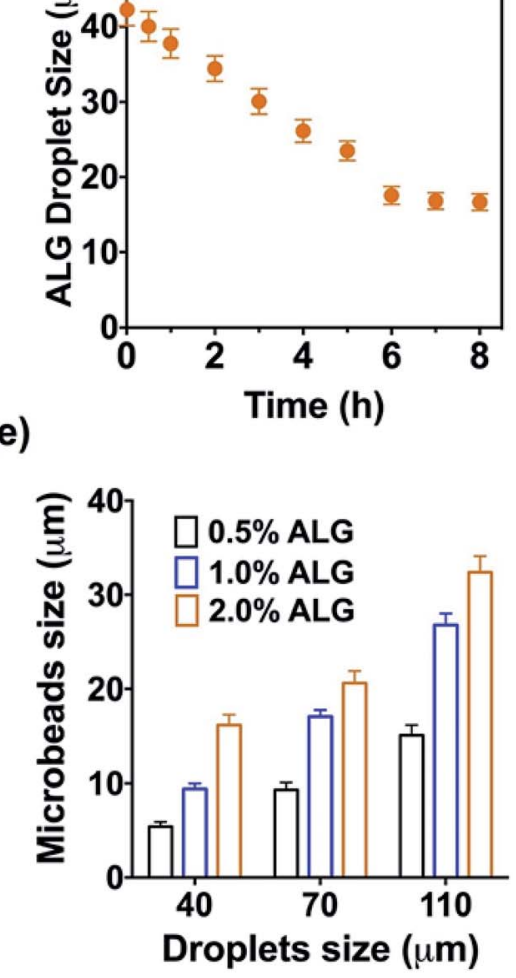

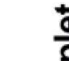

\section{(e)}

(c)
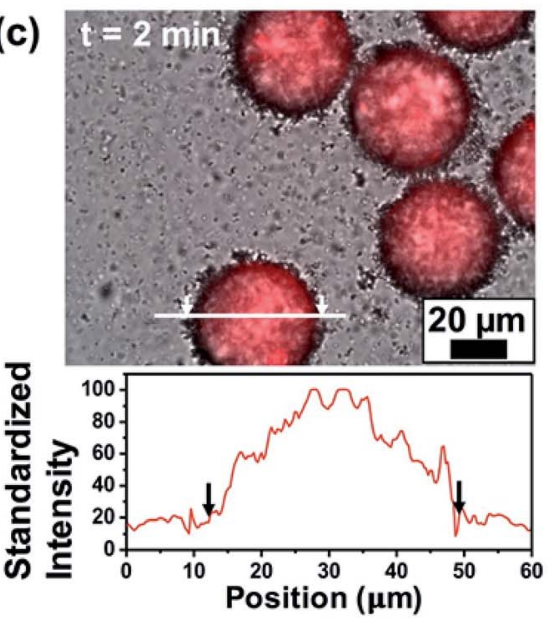

(d)
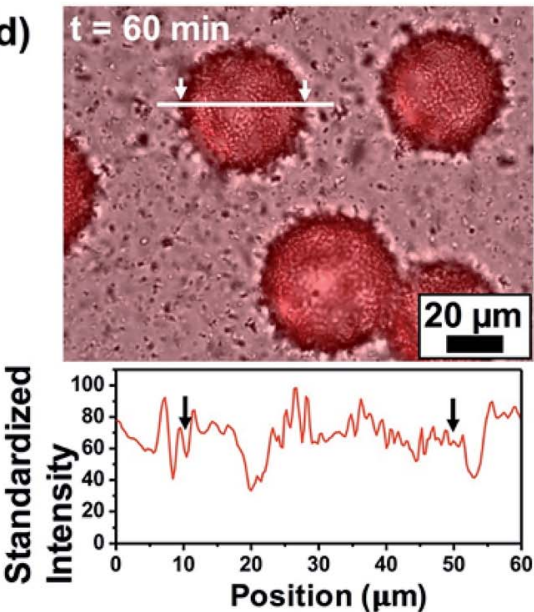

$$
\text { (1) }
$$

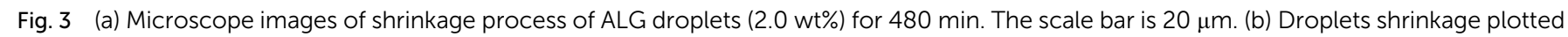

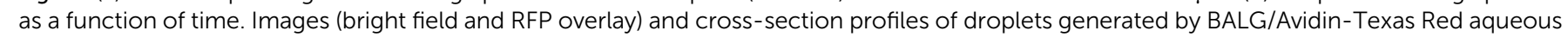

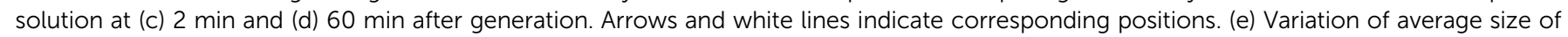
shrunken-MB plotted as a function of the original droplets size.

concentrations. The initial droplet sizes and the sizes of shrunken-MB after $8 \mathrm{~h}$ gelation were summarized in Fig. 3 (e). For the initial droplet size of $40 \mu \mathrm{m}$ with ALG concentration of $0.5 \%, 1.0 \%$ and $2.0 \%$, the final mean sizes of shrunken-MB were 5.8, 9.2 and $16 \mu \mathrm{m}$, respectively. The corresponding shrinkage ratios (defined as initial size/final size) decreased from 6.90 to 4.35 to 2.5. The similar trend was also demonstrated for the initial droplet size of 70 and $110 \mu \mathrm{m}$. Previous studies have shown that the smallest size of droplets that can generated by microfluidic emulsification is largely determined by the orifice dimension of the device. For example, a $10 \mu \mathrm{m}$ by $10 \mu \mathrm{m}$ orifice was optimized to generate ALG droplets with10 $\mu \mathrm{m}$ size. ${ }^{20}$ In general, such small orifice requires a precise microfabrication process; additionally, the device also suffers from operational issues such as clogging and low throughput. In our study, monodispersed and structure uniform microbeads with sizes as small as $5 \mu \mathrm{m}$ were achieved by generating droplets in a microfluidic device with $40 \mu \mathrm{m}$ orifice, followed by the post shrinkage process. By controlling the ALG concentration and initial droplet sizes, 5-30 $\mu \mathrm{m}$ ALG MB were prepared using the same microfluidic device. The polydispersity (CV, coefficient of variance, defined by standard deviation of the MB size divided by the mean MB size) of the final ALG MB with sizes range from 5-30 $\mu \mathrm{m}$ was 4.5 to $8.9 \%$.
Based on the observations described above, the mechanism of droplet shrinkage and ALG shrunken-MB/satellites formation during the gelation process was proposed in Fig. 4. Specifically, at the beginning of gelation, a thin layer of ALG-rich network was formed at the oil/water interface when ALG droplets were expose to mineral oil containing low concentration of $\mathrm{Ca}^{2+}$ ions. During the course of gelation and with more $\mathrm{Ca}^{2+}$ ions diffusion into the ALG-rich network layer, a gelation-induced phase separation occurred and the crosslinked ALG-rich domains were precipitated at the oil/water interface. ${ }^{32,33}$ However, since the $\mathrm{Ca}^{2+}$ ion concentration was too low to fully solidify the precipitated domains, those domains rapidly converted to spherical shapes to minimize the interfacial energy and eventually, transformed to ALG satellites. The gelation process stopped after a few hours when $\mathrm{Ca}^{2+}$ ions reached equilibrium in both oil and water phase, and the original ALG droplet transformed to one shrunken ALG MB and large numbers of ALG satellites. Previously, Zhang et al. reported the formation of ALG MB (40-200 $\mu \mathrm{m}$ in size) with "skinning and grain" structure by diffusion-controlled gelation of ALG droplets in undecanol containing $\mathrm{CaI}_{2}{ }^{13}$ In that work, microgels with a uniform structure were formed and no satellites were observed, which may because: (1) the concentration of $\mathrm{Ca}^{2+}$ ions in undecanol was high and diffusion of $\mathrm{Ca}^{2+}$ ions to the center of the droplet 


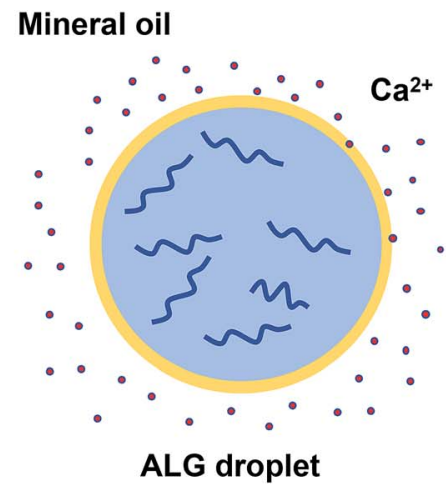

Beginning of gelation

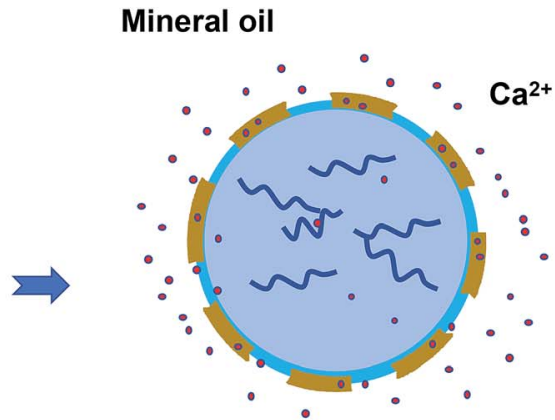

ALG droplet

During the course of gelation

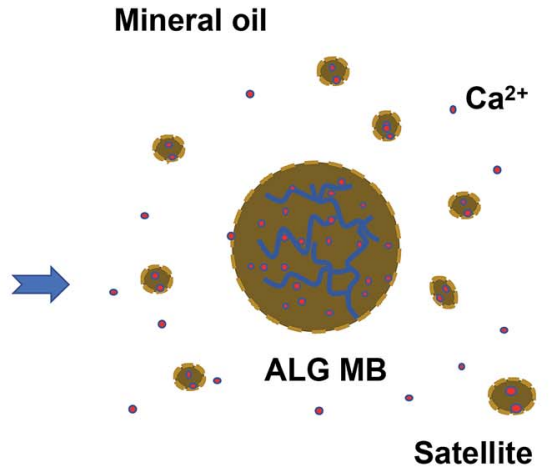

Completion of gelation

Fig. 4 Schematics of ALG droplet shrinkage and formation of ALG shrunken-MB and satellites during the gelation process by diffusion of $\mathrm{Ca}^{2+}$ from mineral oil phase to droplet phase.

was fast enough to suppress phase separation, and (2) solubility of ALG-rich domains in undecanol was probably higher than that in mineral oil. More in-depth study on the mechanism is currently ongoing.

\section{Surface and internal structure of the ALG MB}

To analyze the surface morphology and internal microstructure of the crosslinked ALG MB, we investigated the MB by Scanning Electron Microscope (SEM) and Transmission Electron Microscope (TEM), respectively. As shown in Fig. $5 \mathrm{a}$ and b, microbeads made from $0.5 \mathrm{wt} \%$ ALG solution presented rougher surfaces compared to MB made from $2.0 \mathrm{wt} \%$ ALG solution. Some unmoved satellites can also be found in the SEM images. Additionally, from the TEM images, it is clear that MB made from $2.0 \mathrm{wt} \%$ ALG solution have a denser internal structure, compared to that of the MB made from 0.5\% ALG solution. It is expected that $\mathrm{MB}$ with denser internal structural (smaller porosity) may result in higher stiffness, while MB with a looser internal structural (larger porosity) may result in lower stiffness. Since cells respond differently on substrates with different stiffness, the ALG MB reported in this work may find some potential application in culturing and guiding differentiation of stem cells. ${ }^{34,35}$
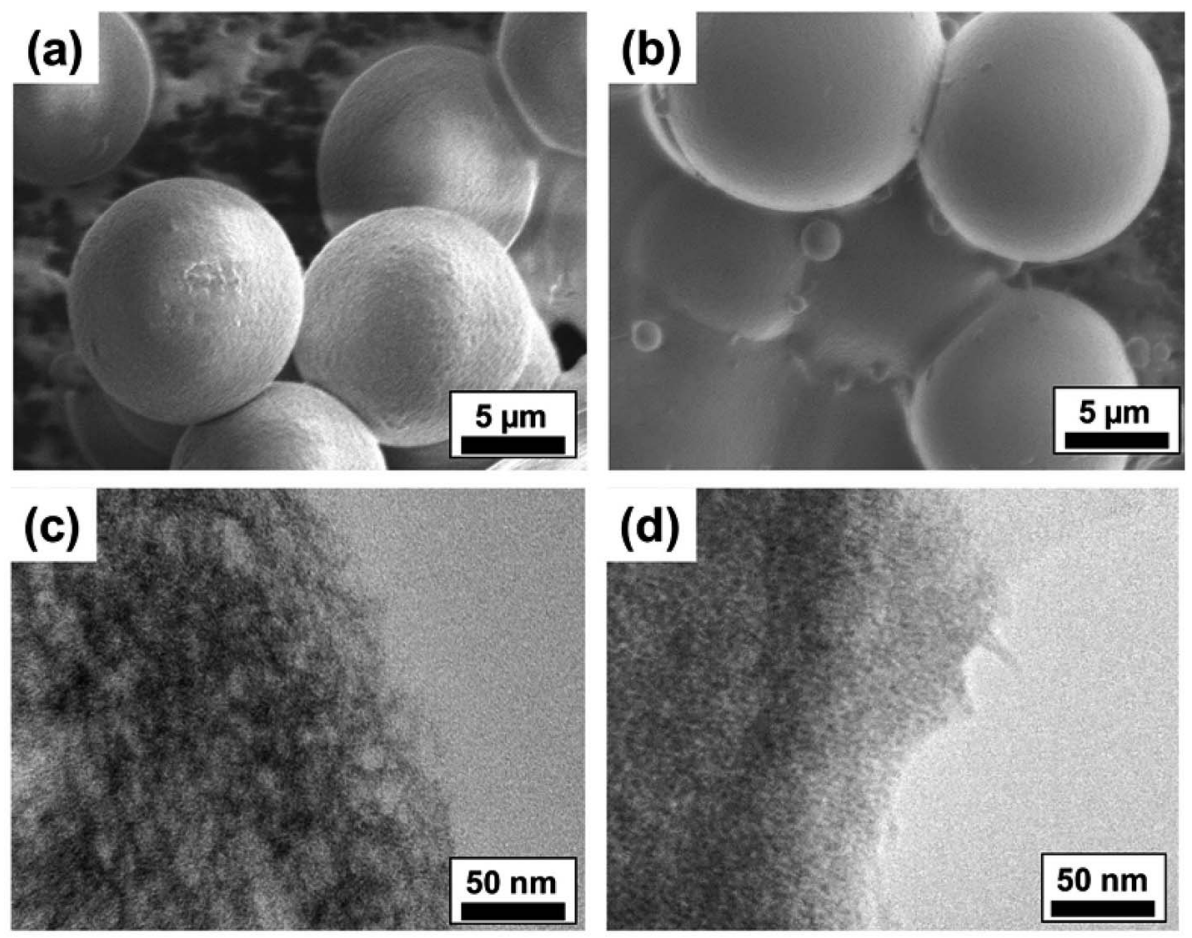

Fig. 5 SEM images of the surface morphology of ALG MB generated from (a) 0.5 wt\% and (b) 2.0 wt\% ALG solutions. TEM images of internal microstructure of ALG MB generated from (c) $0.5 \mathrm{wt} \%$ and (d) $2.0 \mathrm{wt} \%$ ALG solutions. 


\section{Surface modification of ALG MB}

Layer-by-layer ( $\mathrm{LbL}$ ) assembly is a versatile and pervasive surface modification technique where a broad range of materials, such as polymers, DNA, proteins, drug molecules, lipids, and nanoparticles, are used to functionalize substrates. ${ }^{36-40}$ To validate the capability of LbL to form coating on ALG MB, we have conducted the assembly of fluorescein-labeled PAH (FPAH) and biotinylated ALG (BALG) to form a LbL nanofilm on the surface of ALG MB. As the surface of unmodified ALG MB was negatively charged, positively charged FPAH was first used. A thorough washing was performed to remove loosely bound FPAH molecules, then BALG was absorbed and washed after absorption. This process was repeated five times to achieve a thin coating with thickness of approximately $60 \mathrm{~nm}$ (measured from films prepared on a silicon wafer using same experimental conditions). As shown in Fig. 6a, green fluorescent signal was observed, indicating the successful assembly of PAH in the coating. Then, the surface was conjugated with Avidin-Texas Red solution, and the red fluorescent signal confirmed that the biotin moieties was absorbed in the LbL film, which was linked to the dye-labeled Avidin molecules. Furthermore, we have successfully conducted LbL assembly of BALG/PARG films under different experimental conditions and Fig. 6b summarizes the thickness of all the LbL films. Regardless of the molecular weight of PARG used as polycations, a BALG solution with a higher $\mathrm{pH}$ always gives thinner films, which can be explained by the charge density of ALG molecules. Particularly, as alginate has a $\mathrm{p} K_{\mathrm{a}}$ close to 3.5 , ALG polymer in a $\mathrm{pH} 7.0$ solution is more charged than that in a $\mathrm{pH} 4.4$ solution, resulting in a more compact and thinner film due to stronger ionic interactions between PAH and ALG polymers. ${ }^{41}$

\section{Degradation of the surface coating of $[\text { PARG/BALG }]_{5}$ on ALG MB}

One unique advantage of coating the surface of MB with ALGbased LbL nanofilm is that the film can be degraded using a dilute aqueous solution of alginate lyase. This feature can be designed for tethering function molecules or controlled release of bioreagents for future 3D culture systems. Films were labeled with a red fluorescent dye Texas Red for facile monitoring of film functionalization and degradation. Film degradation was due to hydrolysis of ALG molecules by enzymatic cleavage, indicated by the decreased film fluorescence intensity after exposing the MB to the enzyme solution. ${ }^{30}$ We quantified both the initial film brightness and the decreased brightness after degradation. As shown in Fig. 6c, an intense fluorescent signal was observed before degradation, suggesting that the surface of ALG MB was modified with BALG film. A brighter film was expected to contain higher concentrations of bonded avidin molecules, which would allow greater conjugation of biotin labeled bioreagents for later applications. After 30 min degradation in the ALG lyase solution, the fluorescent intensity on the MB surface was dramatically decreased (Fig. 6d), indicating a quick and effective decomposition of the PARG/BALG film. Images of the film fluorescence intensity before and after degradation were recorded and analyzed by ImageJ, and the results indicated that approximately $70 \%$ of film was degraded in the first $10 \mathrm{~min}$ and further degradation was not significant. Although alginate lyase was known to only cleave glycosidic bonds in the backbone of alginate chains, PARG molecular was also dissociated from the film surface along with ALG during degradation. As a result, the degradation of the film did not change its elemental composition of major elements (i.e. carbon, oxygen, hydrogen and nitrogen). ${ }^{30}$

\section{D culture of ALB MB with fibroblast cells}

Finally, we demonstrated the biocompatibility of PARG/BALG coated MB by culturing them with NIH-3T3 cells expressing green fluorescent proteins (NIH-3T3-eGFP). Meanwhile, a bulk flat PARG/BALG-coated ALG hydrogel was carried out as a control experiment for $2 \mathrm{D}$ conditions. In both cases the last layer of the film was capped with BALG polymer. As shown in Fig. 7, NIH-3T3-eGFP cells were evenly dispersed in ALG
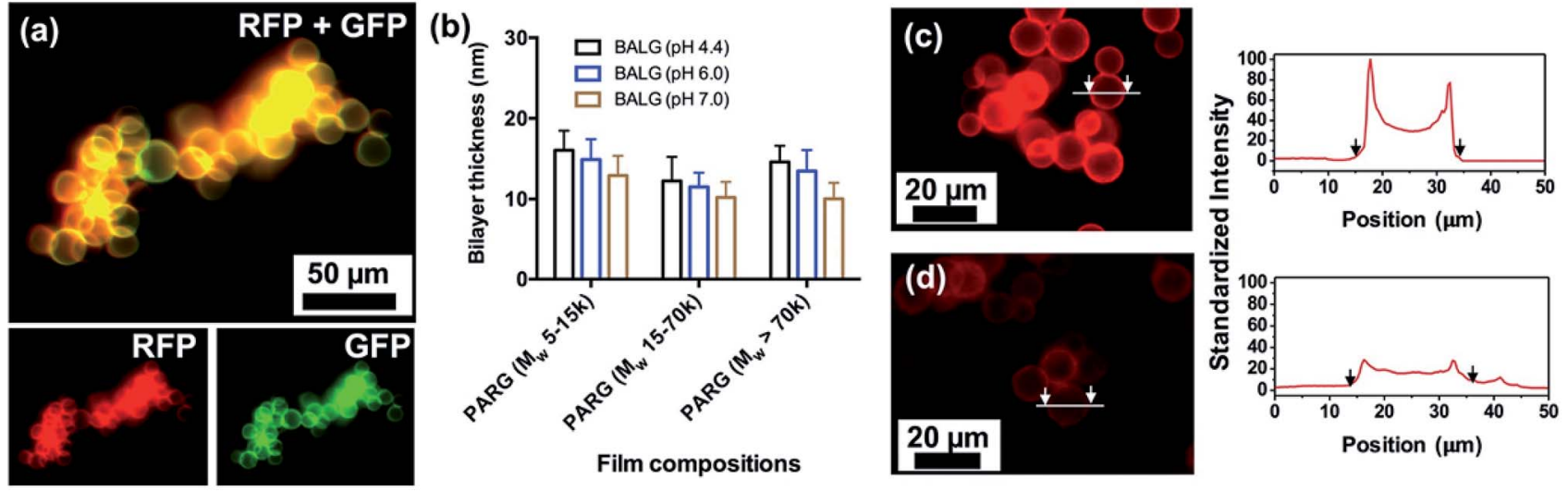

Fig. 6 (a) Fluorescent images of ALG MB coated with five bilayers of fluorescein-labeled PAH (FPAH) and biotinylated ALG (BALG) film followed by conjugating with Avidin-Texas Red. (b) Thickness of a group of poly-L-arginine (PARG)/BALG films prepared on silicon wafer using different assembly conditions. Fluorescent images and cross-section profile of Texas Red fluorescent signal on ALG MB conjugated with five layers of PARG/BALG film followed by conjugating of Avidin-Texas Red, before (c) and after (d) degradation. Arrows and white lines indicate corresponding positions. 

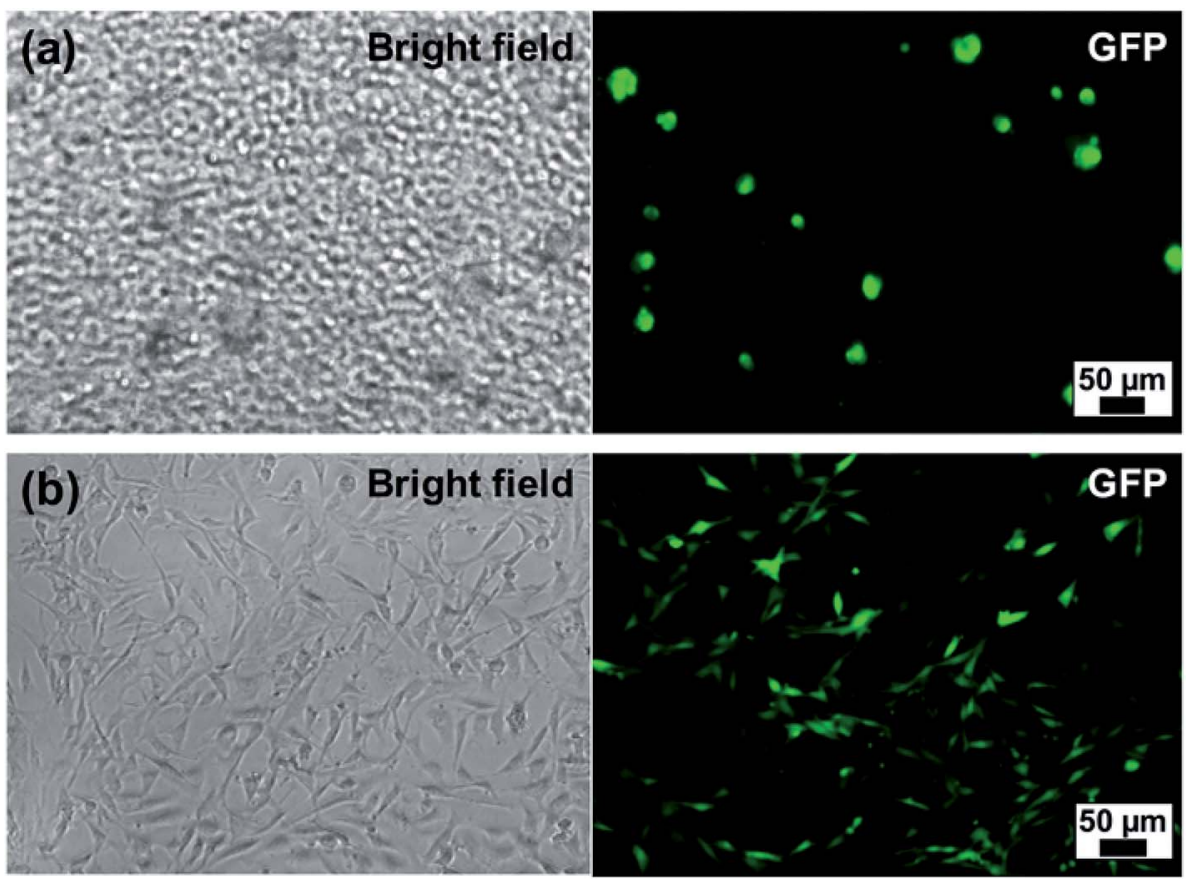

Fig. $7 \mathrm{NIH}$-3T3-eGFP cells cultured at day 4 with (a) ALG MB (2.0 wt\%) and (b) ALG bulk hydrogel. Cell : microbeads = $1: 4$ with cell concentration $10^{5}$ per $\mathrm{mL}$.

microbeads or on ALG hydrogel. Cells cultured in 3D and 2D condition have demonstrated completely different morphology, similar to previously reported works. ${ }^{19,42}$ The cells seeded on 2D bulk hydrogel adhered on the surface and demonstrated typical spreading shapes, while cells cultured in 3D ALG MB comprising single cell, cell-cell cluster and cell-microbead aggregates maintained nearly spherical shapes. The cell clusters ranged in size from 20-50 microns, containing a few to tens of cells. Live/Dead cell viability testing kit was used to determine cell viability for cells cultured, and in both cases $85 \%$ of viability was achieved at day 7 , indicating good biocompatibility of the PARG/BALG film with NIH-3T3-eGFP cells. However, we did not observe noticeable proliferation in the $3 \mathrm{D}$ condition, as cells formed aggregates and did not adhere to the surface of MPs. Further modification of MP surface with adhesion molecules (such as RGDs) or adding growth factor into the culture maybe able to trigger proliferation. Our results suggested the 3D ALG MB culture platform can be used as good blank slate for future studies. Methods for further tethering functional groups on the surface of $\mathrm{MB}$ or integrating additional MB functionalities have been summarized elsewhere. ${ }^{1}$

\section{Conclusions}

In this study, monodispersed ALG microbeads with targeting size of $5-30 \mu \mathrm{m}$ were fabricated by shrinking droplets from original diameter of $40-110 \mu \mathrm{m}$, generated by a microfluidic flow focusing device. The sizes, surface morphology and inner microstructure of the final $\mathrm{MB}$ were controlled by the initial concentration of ALG polymer and the original droplet sizes. Droplet shrinkage was triggered by ALG gelation with $\mathrm{Ca}^{2+}$ and the formation of submicron size satellites due to polymerization-induced phase separation. The described method is capable of generating various cell-size ALG microbeads using the same device, is not limited by sophisticated microfabrication techniques, and avoids microchannel clogging issues. The surface of the prepared ALG MBs can be modified by a layer-by-layer (LbL) assembly method, providing the potential of adding functional molecules for future specified studies. Furthermore, using NIH-3T3-eGFP cells as a model cell for co-culturing with the film-modified ALG MB, we demonstrated good biocompatibility of the 3D culture platform.

\section{Conflicts of interest}

There are no conflicts to declare.

\section{Acknowledgements}

W. L. acknowledges funding support from New Faculty Startup Funds from Texas Tech University and the Cancer Prevention \& Research Institute of Texas (CPRIT) under Grant RP170817. Z. D. is partially supported by CPRIT. D. Y. acknowledges the funding from Henan Natural Science Foundation of China (No. 162102310272). J. C. acknowledges the funding from Henan Natural Science Foundation of China (No. 142102310391).

\section{References}

1 C. C. Ahrens, Z. Dong and W. Li, Engineering cell aggregates through incorporated polymeric microparticles, Acta Biomater., 2017, 62, 64-81. 
2 J. Thiele, Y. Ma, S. M. C. Bruekers, S. Ma and W. T. S. Huck, 25th anniversary article: designer hydrogels for cell cultures: a materials selection guide, Adv. Mater., 2014, 26, 125-148.

3 L. R. Shang, Y. Cheng and Y. J. Zhao, Emerging Droplet Microfluidics, Chem. Rev., 2017, 117(12), 7964-8040.

$4 \mathrm{M}$. George and T. E. Abraham, Polyionic hydrocolloids for the intestinal delivery of protein drugs: alginate and chitosan - a review, J. Controlled Release, 2006, 114(1), 1-14.

5 Y. Y. Zhao, M. T. Carvajal, Y. Y. Won and M. T. Harris, Preparation of calcium alginate microgel beads in an electrodispersion reactor using an internal source of calcium carbonate nanoparticles, Langmuir, 2007, 23(25), 12489-12496.

6 A. Schoubben, P. Blasi, S. Giovagnoli, C. Rossi and M. Ricci, Development of a scalable procedure for fine calcium alginate particle preparation, Chem. Eng. J., 2010, 160(1), 363-369.

7 I. Wands, D. E. T. Shepherd and D. W. L. Hukins, Viscoelastic properties of composites of calcium alginate and hydroxyapatite, J. Mater. Sci.: Mater. Med., 2008, 19(6), 2417-2421.

8 S. Leick, S. Henning, P. Degen, D. Suter and H. Rehage, Deformation of liquid-filled calcium alginate capsules in a spinning drop apparatus, Phys. Chem. Chem. Phys., 2010, 12(12), 2950-2958.

9 M. Becerra, B. Baroli, A. M. Fadda, J. B. Mendez and M. I. G. Siso, Lactose bioconversion by calcium-alginate immobilization of Kluyveromyces lactis cells, Enzyme Microb. Technol., 2001, 29(8-9), 506-512.

10 R. Vreeker, L. B. Li, Y. P. Fang, I. Appelqvist and E. Mendes, Drying and Rehydration of Calcium Alginate Gels, Food Biophys., 2008, 3(4), 361-369.

11 R. Lagoa and J. R. Rodrigues, Kinetic analysis of metal uptake by dry and gel alginate particles, Biochem. Eng. J., 2009, 46(3), 320-326.

12 T. D. Dang and S. W. Joo, Preparation of tadpole-shaped calcium alginate microparticles with sphericity control, Colloids Surf., B, 2013, 102, 766-771.

13 H. Zhang, E. Tumarkin, R. Peerani, Z. Nie, R. M. A. Sullan, G. C. Walker and E. Kumacheva, Microfluidic production of biopolymer microcapsules with controlled morphology, J. Am. Chem. Soc., 2006, 128(37), 12205-12210.

14 A. G. Hati, D. C. Bassett, J. M. Ribe, P. Sikorski, D. A. Weitz and B. T. Stokke, Versatile, cell and chip friendly method to gel alginate in microfluidic devices, Lab Chip, 2016, 16(19), 3718-3727.

15 C. H. Choi, J. H. Jung, Y. W. Rhee, D. P. Kim, S. E. Shim and C. S. Lee, Generation of monodisperse alginate microbeads and in situ encapsulation of cell in microfluidic device, Biomed. Microdevices, 2007, 9(6), 855-862.

16 L. Capretto, S. Mazzitelli, C. Balestra, A. Tosi and C. Nastruzzi, Effect of the gelation process on the production of alginate microbeads by microfluidic chip technology, Lab Chip, 2008, 8(4), 617-621.

17 C. J. Martinez, J. W. Kim, C. W. Ye, I. Ortiz, A. C. Rowat, M. Marquez and D. Weitz, A Microfluidic Approach to Encapsulate Living Cells in Uniform Alginate Hydrogel Microparticles, Macromol. Biosci., 2012, 12(7), 946-951.
18 J. D. Wan, Microfluidic-Based Synthesis of Hydrogel Particles for Cell Microencapsulation and Cell-Based Drug Delivery, Polymers, 2012, 4(2), 1084-1108.

19 W. Li, S. Lee, M. Ma, S. M. Kim, P. Guye, J. R. Pancoast, D. G. Anderson, R. Weiss, R. T. Lee and P. T. Hammond, Microbead-based biomimetic synthetic neighbors enhance survival and function of rat pancreatic $\beta$-cells, Sci. Rep., 2013, 3, 1-10.

20 M. Werner, S. B. G. Blanquer, S. P. Haimi, G. Korus, J. W. C. Dunlop, G. N. Duda, D. W. Grijpma and A. Petersen, Surface Curvature Differentially Regulates Stem Cell Migration and Differentiation via Altered Attachment Morphology and Nuclear Deformation, Adv. Sci., 2017, 4(2), 1600347.

21 S. Utech, R. Prodanovic, A. S. Mao, R. Ostafe, D. J. Mooney and D. A. Weitz, Microfluidic Generation of Monodisperse, Structurally Homogeneous Alginate Microgels for Cell Encapsulation and 3D Cell Culture, Adv. Healthcare Mater., 2015, 4(11), 1628-1633.

22 H. Hirama, T. Kambe, K. Aketagawa, T. Ota, H. Moriguchi and T. Torii, Hyper Alginate Gel Microbead Formation by Molecular Diffusion at the Hydrogel/Droplet Interface, Langmuir, 2013, 29(2), 519-524.

23 K. S. Huang, Y. S. Lin, C. H. Yang, C. W. Tsai and M. Y. Hsu, In situ synthesis of twin monodispersed alginate microparticles, Soft Matter, 2011, 7(14), 6713-6718.

24 D. Saeki, S. Sugiura, T. Kanamori, S. Sato and S. Ichikawa, Formation of monodisperse calcium alginate microbeads by rupture of water-in-oil-in-water droplets with an ultrathin oil phase layer, Lab Chip, 2010, 10(17), 2292-2295.

25 W. Li, J. Greener, D. Voicu and E. Kumacheva, Multiple modular microfluidic (M3) reactors for the synthesis of polymer particles, Lab Chip, 2009, 9, 2715-2721.

26 W. Li, H. H. Pharn, Z. Nie, B. MacDonald, A. Guenther and E. Kumacheva, Multi-step microfluidic polymerization reactions conducted in droplets: The internal trigger approach, J. Am. Chem. Soc., 2008, 130(30), 9935-9941.

27 S. A. Castleberry, W. Li, D. Deng, S. Mayner and P. T. Hammond, Capillary Flow Layer-by-Layer: A Microfluidic Platform for the High-Throughput Assembly and Screening of Nanolayered Film Libraries, ACS Nano, 2014, 8(7), 6580-6589.

28 Z. Dong, C. C. Ahrens, D. Yu, Z. Ding, H. Lim and W. Li, Cell Isolation and Recovery Using Hollow Glass Microspheres Coated with Nanolayered Films for Applications in Resource-Limited Settings, ACS Appl. Mater. Interfaces, 2017, 9(18), 15265-15273.

29 Z. Dong, D. Yu, Q. Liu, Z. Ding, V. J. Lyons, D. Pappas, X. Liu and W. Li, Enhanced Capture and Release of Circulating Tumor Cells Using Hollow Glass Microspheres with Nanostructured Surface, Nanoscale, 2018, 10(35), 1679516804.

30 Z. Dong, L. Tang, C. C. Ahrens, Z. Ding, V. Cao, S. Castleberry, J. Yan and W. Li, A benchtop capillary flow layer-by-layer (CF-LbL) platform for rapid assembly and screening of biodegradable nanolayered films, Lab Chip, 2016, 16(23), 4601-4611. 
31 W. Li, E. Young, P. Garstecki, C. A. Simmons and E. Kumacheva, Simultaneous Microfluidic Generation of Droplets with Different Dimensions, Soft Matter, 2008, 4(2), 258-262.

32 N. G. Podosenova and V. P. Budtov, Phase separation in a polymerizing polymer-monomer system, Polym. Sci. U.S.S.R., 1988, 30(11), 2428-2434.

33 H. M. J. Boots, J. G. Kloosterboer, C. Serbutoviez and F. J. Touwslager, Polymerization-induced phase separation.1. Conversion-phase diagrams, Macromolecules, 1996, 29(24), 7683-7689.

$34 \mathrm{Y}$. Shao and J. Fu, Integrated Micro/Nanoengineered Functional Biomaterials for Cell Mechanics and Mechanobiology: A Materials Perspective, Adv. Mater., 2014, 26(10), 1494-1533.

35 Y. Shao, J. Sang and J. Fu, On human pluripotent stem cell control: the rise of $3 \mathrm{D}$ bioengineering and mechanobiology, Biomaterials, 2015, 52, 26-43.

36 P. T. Hammond, Engineering materials layer-by-layer: challenges and opportunities in multilayer assembly, AIChE J., 2011, 57(11), 2928-2940.
37 J. Borges and J. F. Mano, Molecular Interactions Driving the Layer-by-Layer Assembly of Multilayers, Chem. Rev., 2014, 114(18), 8883-8942.

38 W. J. Tong, X. X. Song and C. Y. Gao, Layer-by-layer assembly of microcapsules and their biomedical applications, Chem. Soc. Rev., 2012, 41(18), 6103-6124.

39 J. J. Richardson, M. Bjornmalm and F. Caruso, Technologydriven layer-by-layer assembly of nanofilms, Science, 2015, 348(6233), aaa2491.

40 J. J. Richardson, J. Cui, M. Björnmalm, J. A. Braunger, H. Ejima and F. Caruso, Innovation in Layer-by-Layer Assembly, Chem. Rev., 2016, 116(23), 14828-14867.

41 W. Li, E. Reátegui, M.-H. Park, S. Castleberry, J. Z. Deng, B. Hsu, S. Mayner, A. E. Jensen, L. V. Sequist, S. Maheswaran, D. A. Haber, M. Toner, S. L. Stott and P. T. Hammond, Biodegradable nano-films for capture and non-invasive release of circulating tumor cells, Biomaterials, 2015, 65, 93-102.

42 A. B. Bernard, R. Z. Chapman and K. S. Anseth, Controlled Local Presentation of Matrix Proteins in Microparticle-Laden Cell Aggregates, Biotechnol. Bioeng., 2014, 111(5), 1028-1037. 